\title{
Longitudinal Muscle Dysfunction in Achalasia Esophagus and Its Relevance
}

\author{
Ravinder K Mittal, ${ }^{*}$ Su Jin Hong and Valmik Bhargava \\ Division of Gastroenterology, Department of Medicine, San Diego VA Health Care System and University of California, San Diego, CA, USA
}

Muscularis propria of the esophagus is organized into circular and longitudinal muscle layers. Goal of this review is to summarize the role of longitudinal muscle in physiology and pathophysiology of esophageal sensory and motor function. Simultaneous manometry and ultrasound imaging that measure circular and longitudinal muscle contraction respectively reveal that during peristalsis 2 layers of the esophagus contract in perfect synchrony. On the other hand, during transient relaxation of the lower esophageal sphincter (LES), longitudinal muscle contracts independently of circular muscle. Recent studies provide novel insights, i.e., longitudinal muscle contraction of the esophagus induces LES relaxation and possibly descending relaxation of the esophagus. In achalasia esophagus and other motility disorders there is discoordination between the 2 muscle layers. Longitudinal muscle contraction patterns are different in the recently described three types of achalasia identified by high-resolution manometry. Robust contraction of the longitudinal muscle in type II achalasia causes pan-esophageal pressurization and is the mechanism of whatever little esophageal emptying that take place in the absence of peristalsis and impaired LES relaxation. It may be that preserved longitudinal muscle contraction is also the reason for superior outcome to medical/surgical therapy in type II achalasia esophagus. Prolonged contractions of longitudinal muscles of the esophagus is a possible mechanism of heartburn and "angina like" pain seen in esophageal motility disorders and possibly achalasia esophagus. Novel techniques to record longitudinal muscle contraction are on the horizon. Neuro-pharmacologic control of circular and longitudinal muscles is different, which provides an important opportunity for the development of novel pharmacological therapies to treat sensory and motor disorders of the esophagus.

(J Neurogastroenterol Motil 2013;19:126-136)

\section{Key Words}

Esophageal achalasia; Esophageal motility disorders; Muscles; Pain

\section{Introduction}

Esophagus is no exception; similar to the rest of the gastrointestinal tract, outer muscular coat of the esophagus, i.e., muscu- laris propria is organized into an outer longitudinal and an inner circular muscle layers. Longitudinal muscle of the esophagus originate as three bundles from the cricoids cartilage, (1) a main longitudinal muscle bundle that originates from the posterior surface of the cricoids cartilage, (2) an accessory muscle bundle from

Received: February 19, 2013 Revised: March 9, 2013 Accepted: March 10, 2013

(c) This is an Open Access article distributed under the terms of the Creative Commons Attribution Non-Commercial License (http://creativecommons. org/licenses/by-nc/3.0) which permits unrestricted non-commercial use, distribution, and reproduction in any medium, provided the original work is properly cited.

*Correspondence: Ravinder K Mittal, MD

Gastroenterology (111D), San Diego VA Health Care System, 3350, La Jolla Village Drive, San Diego, CA 92161, USA

Tel: +1-858-552-7556, Fax: +1-858-552-4327, E-mail: rmittal@ucsd.edu

Financial support: This work was supported by an NIH (Grant No. R01DK060733).

Conflicts of interest: None.

Author contributions: Ravinder K Mittal designed the experiments, oversaw data acquisition and wrote the manuscript. Su Jin Hong acquired and analyzed data and helped with writing the manuscript. Valmik Bhargava wrote the software for data analysis, designed the experiments, created figures, and wrote and edited the manuscript. 
Longitudinal muscles of the esophagus
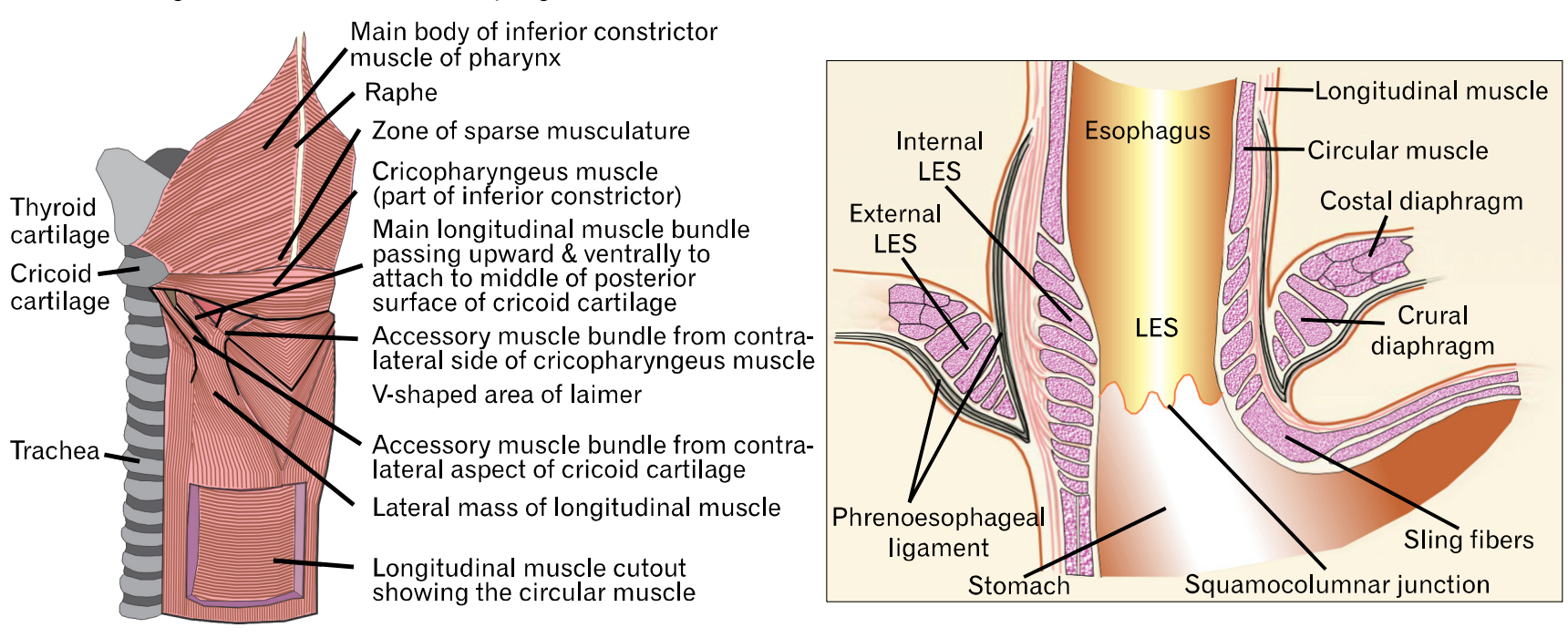

Figure 1. Anatomy of esophagus longitudinal muscle. LES, lower esophageal sphincter. Re-drawn from a drawing by Frank Netter, MD (left) and from Mittal and Balaban (The esophagogastric junction. N Engl J Med 1997;336:924-932).

the posterior-lateral surface of cricoids cartilage and (3) an accessory muscle bundle from the contra lateral surface of the cricopharyngeus muscle (Fig. 1). Muscle bundles from the 2 sides quickly fan out to surround the entire circumference of the esophagus, leaving only a small triangular space, area of Laimer at the most cranial end of the esophagus. At the caudal end, fibers of longitudinal muscle are inserted into the circular muscle bundles of the lower esophageal sphincter (LES) and some continue onto the stomach. Not much has been made of the significance of orientation of muscle fibers in peristalsis and bolus propulsion because it is generally assumed that circumferential occlusion of the lumen caused by circular muscle contraction and its progression in a sequential manner, aboral or oral direction (peristalsis or reverse peristalsis) causes bolus propulsion and retropulsion respectively.

Longitudinal muscles of the muscularis propria comprise 50 percent of the muscle mass of the esophagus. Advances in esophageal manometry, including high-resolution manometry (HRM) have made it easier to study circular muscle function of the esophagus. Large number of publications and consensus group has made it possible to charter "Chicago classification of esophageal motility disorders." ${ }^{1}$ However, Chicago classification and HRM in the current format provides no information on the longitudinal muscles of the esophagus.

A major problem in studying longitudinal muscle layer, unlike circular muscle, is the difficulty in measuring its contraction and relaxation. Longitudinal muscle contraction and relaxation causes axial shortening and lengthening of the esophagus respectively, which cannot be monitored by conventional esophageal manometry recordings. Investigators have used strain gauzes, implanted surgically in the long axis of longitudinal muscle layer, in animal experiments to study longitudinal muscle contraction patterns. ${ }^{2}$ Several investigators have also used radio-opaque markers and X-ray fluoroscopy (in humans $s^{3,4}$ and animals ${ }^{5}$ ) to study longitudinal muscle contraction. These studies have resulted in important information; however one cannot perform X-ray fluoroscopy, especially in the humans for extended time periods because of radiation safety issues. During the last 2 decades ultrasound (US) imaging of the esophagus, performed using thin, 1-2 mm diameter intravascular catheters, has yielded key information on the role of longitudinal muscle layer in health and diseases. $^{6,7}$

US imaging to study longitudinal muscle function is based on the law of mass conservation, i.e., axial shortening of the esophagus results in an increases in muscle thickness, which can be quantitated by image analysis. To date, US imaging is the only technique that measures longitudinal muscle contraction at a point location, just like point sensors of the manometry measures circular muscle contraction at a point location. US image analysis is labor intensive, but it allows prolonged recordings which is essential especially for symptom correlation. The methodology to perform US image recordings, as well as data analysis has been described in detail earlier. ${ }^{7}$ More recently, a sensor that utilizes magnet and electromagnetic field effect (based on Hall's effect) 
has been used to study axial motions of the squamo-columnar junction/esophagogastric junction, as an indirect marker of axial shortening of the esophagus. ${ }^{8}$

\section{Patterns of Longitudinal Muscle Contraction in Health and Its Relevance to Bolus Propulsion During Peristalsis}

Manometry studies reveal that during peristalsis a length of the esophagus, rather than a point location of the esophagus, contracts in a sequential fashion from the oral to the aboral end. The length of contracted esophagus at a given time increases as peristalsis moves in the caudal direction, it can be more than $10 \mathrm{~cm}$ in the distal esophagus. Esophageal pressure (due to circular muscle contraction) in the contracted segment is distributed in the form of a bell shaped curve, with peak pressure in the middle. Simultaneous US imaging and manometry shows that longitudinal muscle also contracts in a fashion identical to the circular muscle, i.e., the 2 contract synchronously, peak of 2 muscle contraction occur within 1 second of each other. ${ }^{10}$ Secondary peristalsis or esophageal propulsive force induced by distension of a balloon in the esophagus also induces contraction and relaxation of the 2 muscle layers synchronously, above and below the site of distension respectively. ${ }^{11}$ Transient LES relaxation, a key motor event that allows retrograde transport of gastric content into the esophagus (vomiting, belching and reflux) is associated with a longitudinal muscle contraction pattern that is different from the peristaltic pattern. ${ }^{12,13}$ Longitudinal muscle contracts independently of circular muscle, contraction starts at the caudal end of the esophagus and progresses in the oral direction. Longitudinal muscle contraction during transient lower esophageal sphincter relaxation (TLESR) is significantly stronger in amplitude and longer in duration than during primary peristalsis; mean duration of contraction 23 vs. 6 seconds, respectively. Another distinct longitudinal muscle contraction is seen in association with subthreshold stimulation of the pharynx, which induces LES relaxation that is not as strong as TLESR or swallow-induced LES relaxation. ${ }^{14}$ With subthreshold pharyngeal stimulus longitudinal muscle contracts only at the cranial end of the esophagus, i.e., few centimeters of the esophagus below the upper esophageal sphincter (UES).

Key question is 'what is the significance of longitudinal muscle contraction to bolus propulsion?' From the mechanics point of view, there are 2 major effects of longitudinal muscle contraction on the esophageal wall. It results in thickening and thinning of the 2 muscle layers in the contracted and relaxed segments of the esophagus respectively. ${ }^{7}$ Two muscle layers slide in relationship to each other in the contracted segment. ${ }^{15}$ Contraction of the longitudinal muscle provides 2 distinct mechanical advantages to the esophageal wall; (1) by increasing muscle thickness it reduces wall stress $^{16}$ and compliance of the esophageal wall in the contracted segment. Both of the above properties are crucial for occlusion of the esophageal lumen that is needed for bolus propulsion. (2) On the other hand, in the segment distal to contraction there is axial lengthening and thinning of the esophageal wall that reduces esophageal wall compliance, which is helpful for accommodation of bolus. Another important effect of longitudinal muscle contraction is the lift of LES in the orad direction. Studies show that an axial stretch in the oral direction induces a neurally mediated relaxation of the LES. ${ }^{17} \mathrm{~A}$ mechanical pull in the axial direction induces activation of inhibitory motor neurons located in the myenteric plexus, which releases nitric oxide (NO) to induce LES relaxation. Along the same lines, it is likely that elongation of the esophageal segment, distal to the site of contraction, induces active relaxation or descending relaxation ${ }^{18}$ of the esophagus distal to the contracted segment which is crucial

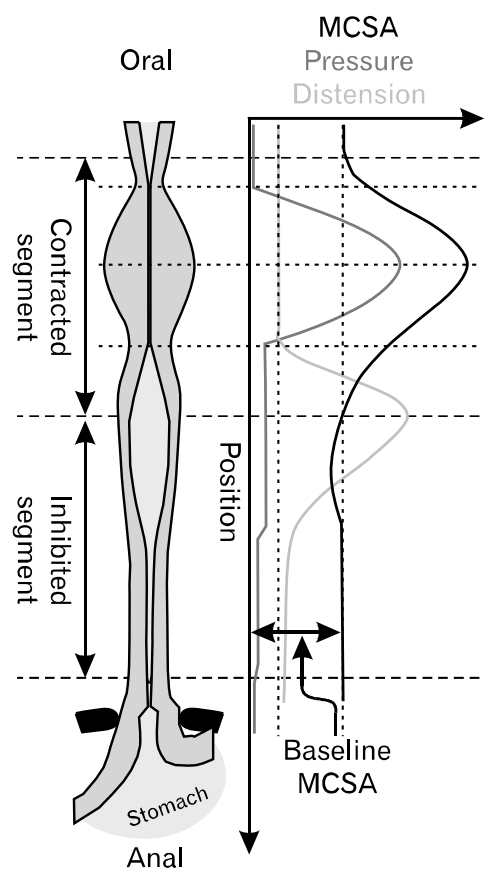

Figure 2. Schematic of contraction and distension during swallow induced peristalsis. Note that the pressure and muscle cross sectional area, surrogate markers of circular and longitudinal muscle contractions respectively, precede distension. The latter marches distally in front of the onset of contraction wave in a peristaltic fashion. MCSA, muscle cross sectional area. Adapted from Abrahao et al. ${ }^{18}$ 
for efficient bolus transport. Relaxation of the esophagus and LES distal to the contracted segment allows contracted and peristaltic segment to propel bolus in the caudal direction against minimal resistance in the relaxed segment (Fig. 2). Peristaltic reflex by definition has 2 components, i.e., ascending contraction and descending relaxation. It seems that longitudinal muscle contraction plays important and distinct roles in the ascending contraction as well as descending relaxation limbs of the peristaltic reflex.

Eosinophilic esophagitis, which is being diagnosed with increasing frequency, is caused by allergy to several food constituents. ${ }^{19}$ Increase in number of eosinophilia (15-20/high power field) in the esophageal mucosa is the hallmark of eosinophilic esophagitis. ${ }^{20}$ Dysphagia is the major symptom in patients with eosinophilic esophagitis and is thought to results from relative narrowing of the esophagus as a result of fibrosis of the esophageal mucosa. Esophageal manometry, which measures circular muscle contraction only, is relatively normal in majority of patients with eosinophilic esophagitis. Interestingly, longitudinal muscle function is significantly impaired in patients with eosinophilic esophagitis as recorded by US imaging. ${ }^{21}$ It manifests as reduced amplitude of contraction, discoordination between the 2 muscle layers and reduced response to a cholinergic agent. It is very possible that the longitudinal muscle dysfunction leads to impaired descending relaxation of the esophagus that is seen as relative narrowing of the esophagus thus causing dysphagia.

Longitudinal muscle contraction can be mediated through 2 different pathways. During peristalsis it is through the vagus nerve and myenteric plexus neurons that project onto the longitudinal muscles. On the other, in the diseased state, i.e., esophagitis, chemicals released from the mucosa may diffuse into longitudinal muscle to induce contraction and possibly relaxation. During peristalsis, neurotransmitters responsible for contraction and relaxation of longitudinal muscle are different from those of circular muscle. With each swallow the circular muscle first hyperpolarizes that results in inhibition, followed by depolarization that causes excitation or contraction. ${ }^{22}$ On the other hand, longitudinal muscles, unlike circular muscles, do not hyperpolarize, and they just depolarize and contract. Another important difference between circular and longitudinal muscle is that NO that induces relaxation in the circular muscle causes contraction in the longitudinal muscle, both of which are mediated via intracellular cyclic GMP. ${ }^{23,24} \mathrm{NO}$ induced contraction of the longitudinal muscle is blocked by cyclooxygenase inhibitor, i.e., indomethacin. In the muscle strip experiments, substance $\mathrm{P}$ along with acetylcholine are the neurotransmitters of longitudinal muscle. ${ }^{25}$ However, in the whole animal preparation the role of $\mathrm{NO}^{26}$ and substance $\mathrm{P}$ in the longitudinal muscle contraction is not clear. Atropine, an anticholinergic agent, almost completely blocks vagus nerve mediated and swallow mediated longitudinal muscle contraction. $^{27}$

It has been demonstrated in both animal as well as human experiments that acid infusion into the esophagus induces longitudinal muscle contraction. ${ }^{28}$ In fact, repeated acid infusion into
A

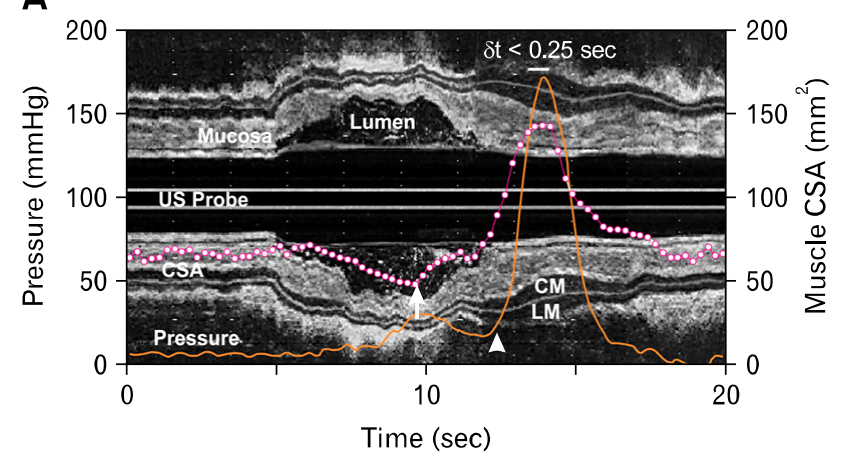

B

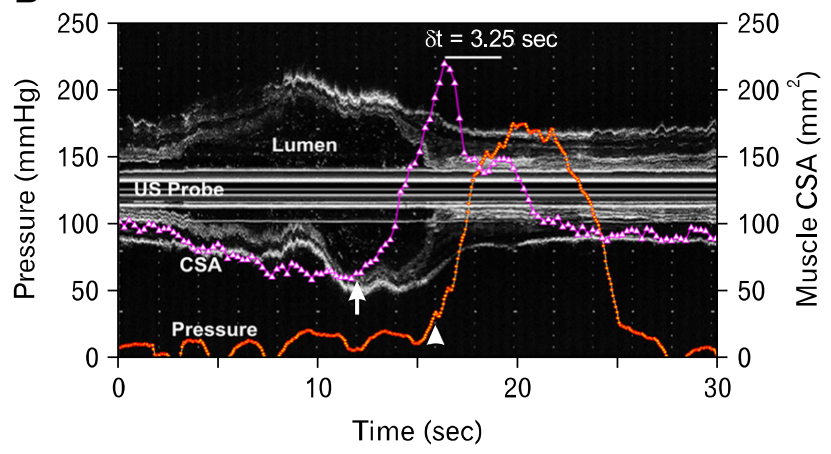

Figure 3. Coordination and discoordination between circular and longitudinal muscle. (A) Relationship between M-mode ultrasound images, muscle cross sectional area (CSA) and the pressure wave in a normal subject. It shows the onset of lumen collapse occurs at the same time as the onset of muscle CSA. Pressure wave occurs at the same time as the first complete collapse of the lumen on the manometric probe. Peak of manometric contraction and peak CSA occurred with a delta-t $\leq 0.5$ seconds during all 28 swallows at the $2 \mathrm{~cm}$ and all 28 swallows at the $10 \mathrm{~cm}$ level. (B) An example of dissociation between circular and longitudinal muscle contraction in a patient with nutcracker esophagus. These recordings were obtained at the $2 \mathrm{~cm}$ above the lower esophageal sphincter. Note the disassociation between the peak pressure and peak muscle CSA. US, ultrasound. Adapted from Jung et al. ${ }^{33}$ 
the esophagus sensitizes and potentiates longitudinal muscle contraction. ${ }^{29}$ In response to acid infusion, mast cells located in the submucosa release number of chemicals, histamine, serotonin and trypsine..$^{30,31}$ These chemicals act on the capsaicin sensitive nerve endings (extrinsic afferent) to release neurokinins and substance $\mathrm{P}$ to stimulate tachykinin receptors on the longitudinal muscles. Longitudinal muscle contraction is impaired in patients with eosinophilic esophagitis ${ }^{21}$ which could possibly be related to the release of an unknown chemical from large number of eosinophils present in the mucosa.

\section{Longitudinal Muscle Contraction in Achalasia Esophagus}

As discussed earlier, during normal peristalsis, longitudinal and circular muscle contract synchronously, the peak of contraction of 2 muscle layers always occurs within 1 second of each other. Increase in cholinergic activity, by an acetylcholinestrase inhibitor (edrophonium) induces disassociation between the 2 muscle layers such that longitudinal muscle contracts prior to the
A

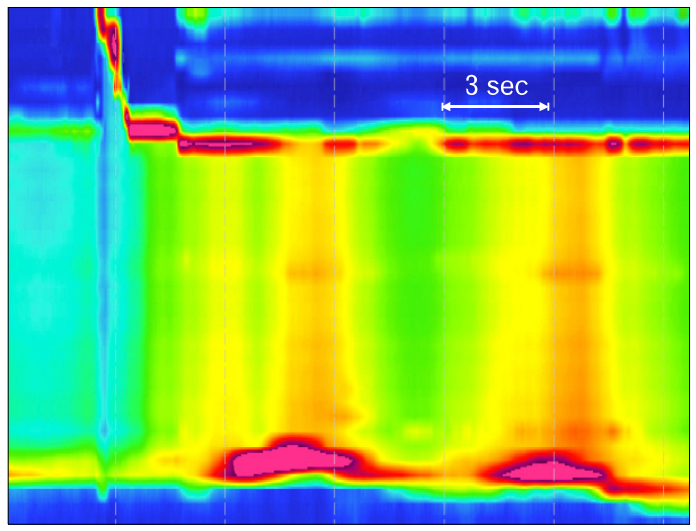

C

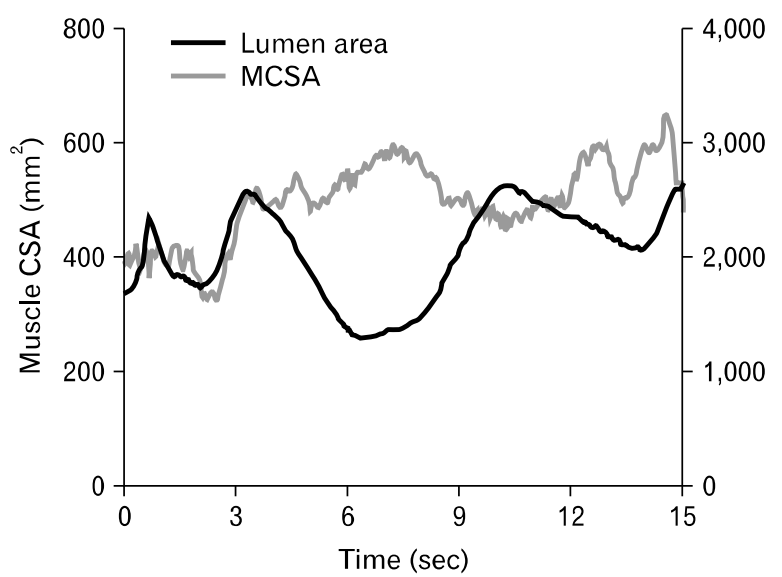

B

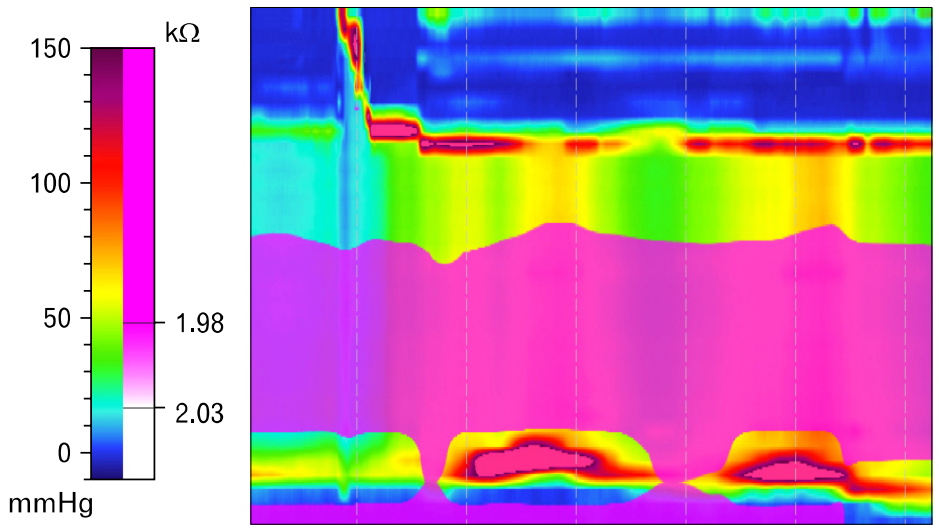

D

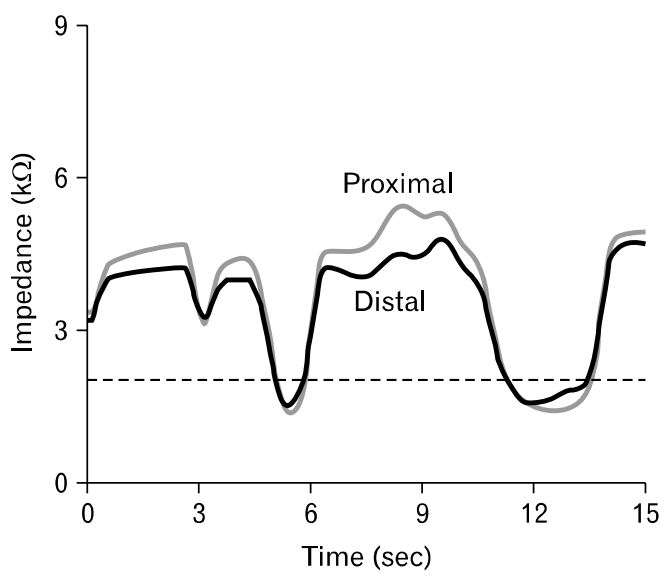

Figure 4. Simultaneous high-resolution manometry (HRM), impedance and ultrasound image recorded changes in esophageal lumen and muscle cross sectional area (CSA) in a patient with type II achalasia. (A) HRM alone, (B) HRM and impedance, (C) changes in muscle CSA and lumen (derived from ultrasound [US] images) and (D) impedance recording from the pair of electrodes above and below the esophagogastric junction (EGJ). Following swallow there is simultaneous pressure wave throughout the length of the esophagus (common cavity wave- panesophageal pressurization). EGJ record shows minimal relaxation to contraction following swallow. Impedance recording shows intermittent flow (seen twice in the record) seen as the development of pink bridge across the EGJ and fall in impedance values to below $2 \mathrm{k} \Omega$ and equal to each other from the pair of electrodes above and below the EGJ. US image derived data show decrease in luminal CSA and increase in muscle CSA during the period of common cavity pressure wave (panesophageal pressurization). Increase in muscle CSA suggests contraction of the longitudinal muscle of the esophagus. Horizontal dashed line represents $2 \mathrm{k} \Omega$ value in panel $\mathrm{D}$. Adapted from Hong et al. ${ }^{36}$ 
circular muscle. $^{32}$ In patients with nutcracker esophagus there is spontaneous disassociation between the timing of 2 muscle layers during peristalsis; contraction of 2 muscles peaks can be separated from each other by several seconds with longitudinal muscle contracting ahead of the circular muscle (Fig. 3). ${ }^{33}$ Interestingly, the disassociation between 2 muscle layers is reduced by atropine (an anticholinergic agent) in patients with nutcracker esophagus. ${ }^{34}$ Latter suggest that the nutcracker esophagus is a hypercholinergic state that manifests as disassociation between the 2 muscle layers (in addition to the higher contraction amplitude and higher contraction duration). An immunohistochemistry study found an increase in expression of muscrinic receptors on the esophageal muscles in patients with nutcracker esophagus. ${ }^{35}$ How does discoordination in the 2 muscle layers cause esophageal symptoms? Even though this is not precisely known, it may be that discoordination between the 2 layers by interfering with descending relaxation of the esophagus leads to relative narrowing of the esophagus that impedes efficient propulsion of the bolus.

Another example of discoordination between the longitudinal and circular muscle layers is seen in achalasia esophagus. ${ }^{36}$ LES relaxation is impaired and there is no peristalsis of circular muscles in the achalasia esophagus. Each swallow result in a simultaneous pressure wave throughout the length of the esophagus in achalasia esophagus, also termed as common cavity pressure wave. It has also been referred to as pressurization of the esophagus. Simultaneous HRM and US imaging reveal that pressurization of the esophagus is actually the results of a distinct motor pattern in which only longitudinal muscle contracts in the distal esophagus (Fig. 4). Contraction of longitudinal muscle causes axial shortening as well as reduction in esophageal cross sectional area. Since esophagus is closed at the 2 ends, by upper and lower esophageal sphincter, reduction in esophageal volume (as a result of axial shortening and reduction in esophageal cross sectional area) leads to a proportional increase in the pressure (Boyles law of physics), referred to as the common cavity pressure or simultaneous pressure wave in the esophagus. It is clear that esophageal pressurization caused by longitudinal muscle contraction is a crucial factor in whatever little esophageal emptying that occurs in achalasia esophagus where LES does not relax and there is no peristalsis in the esophagus.

Based on the swallow-induced pressure patterns in the esophagus, as seen on HRM, Pandolfino et al have categorized achalasia esophagus into three different types. Type I, with minimal pressurization of the esophagus, type II, in which esophageal pressurization is more than $30 \mathrm{mmHg}$, and type III with spastic or high amplitude type of esophageal contraction. ${ }^{37}$ Several studies show that type II achalasia esophagus responds best to medical and surgical treatment (botulinum toxin injection, pneumatic dilation or surgery). ${ }^{37-39}$ It appears that longitudinal muscle con-
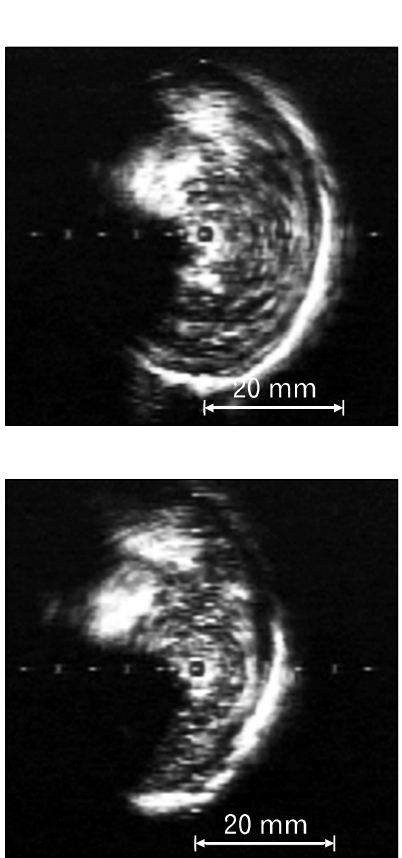

Type I

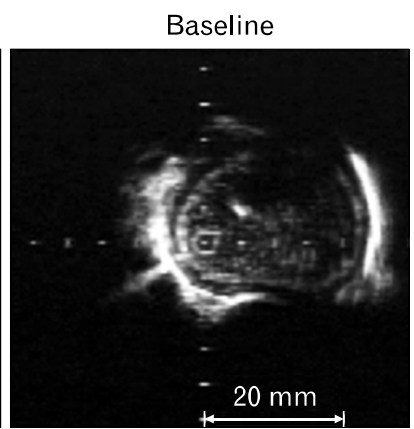

Peak contraction

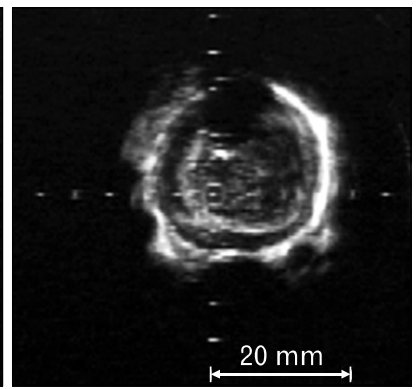

Type II
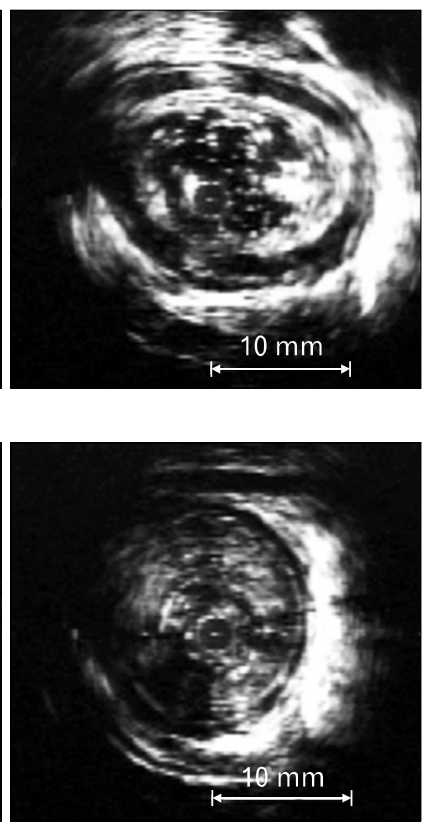

Type III
Figure 5. Ultrasound images of 3 types of achalasia esophagus. These ultrasound images from 3 types of achalasia esophagus show that luminal cross sectional area is largest in type I and muscle is thinnest. On the other hand, luminal size is smallest and thickest in type III achalasia. 
A

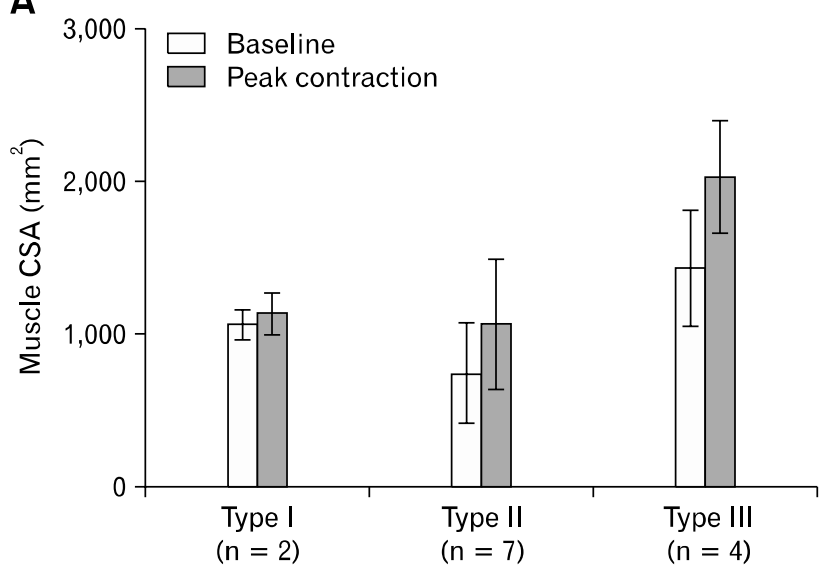

C

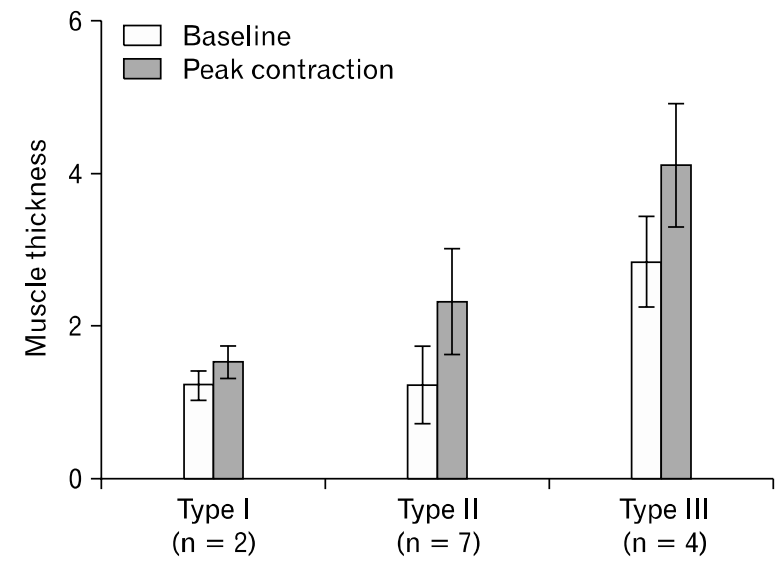

traction patterns are quite different in 3 different types of achalasia. ${ }^{36}$ Type I achalasia shows minimal to no longitudinal muscle contraction of the esophagus. On the other hand, in type II achalasia there is strong longitudinal muscle contraction. In type III achalasia, both circular and longitudinal muscle contract but there is severe discoordination between the 2 muscle layers. Another important difference between 3 types of achalasia is the luminal cross section area and thickness of esophageal muscles at the baseline or in between swallows (Fig. 5 and 6). Luminal cross sectional area is largest in type I and least in type III. On the other hand, esophageal wall and muscle are thinnest in type I (smaller than normal) and thickest in type III, with type II showing esophageal cross sectional area and muscle thickness that are in between type I and type III. According to Laplace law intraluminal pressure is inversely related to the luminal diameter/cross sectional area and directly related to the wall thickness. Therefore, it is not surprising that esophageal pressures are lowest in type I achalasia in which luminal diameter is large and muscle is

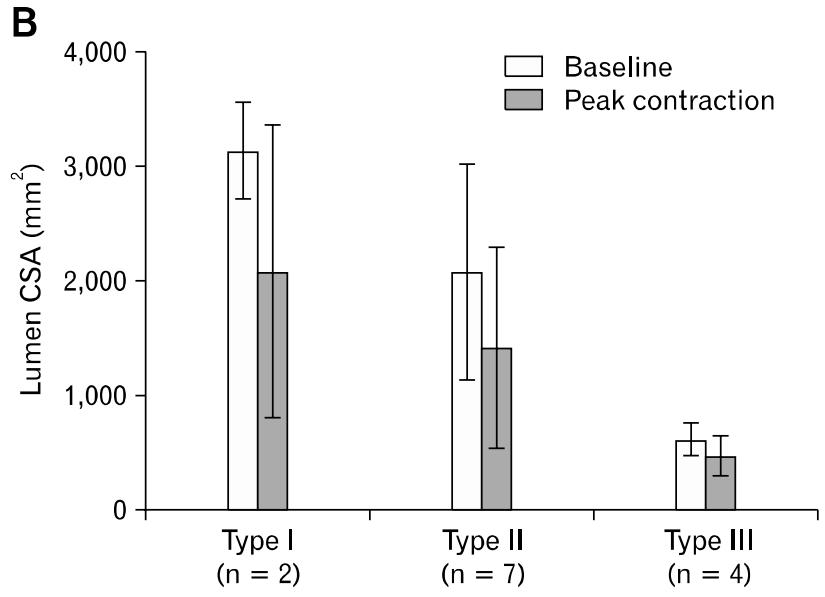

Figure 6. Esophageal muscle cross sectional area (CSA) (A), lumen CSA (B) and muscle wall thickness (C) in 3 types of achalasia esophagus.

thinnest as compared to type II and III achalasia.

Mechanisms of esophageal emptying are also different in three types of achalasia. In type I, esophageal emptying is mainly due to gravity and increase in thoracic pressure caused by unique and unusual maneuvers used by these patients. On the other hand, in type II achalasia, pan-esophageal pressurization related to longitudinal muscle contraction is the main mechanism of intermittent esophageal emptying against a closed LES. Interestingly, at least based on the impedance recordings esophageal transit appears normal (personal observation) in type III achalasia with spastic type of contraction. It is likely that robust longitudinal muscle contraction in type II achalasia esophagus, which is largely responsible for pressurization and emptying of the esophagus, may be the reason for good response to medical/surgical therapy.

Dysphagia is the major symptom in patients with achalasia esophagus; however chest pain ${ }^{40}$ and heartburn ${ }^{41}$ are also reported in more than two-thirds of these patients. Heartburn in achalasia may precede dysphagia and can resolve with onset of 
dysphagia. Even though swallow related LES relaxation is impaired in achalasia but in some patients TLESRs do occur that may cause gastroesophageal acid reflux, which could be the cause of heartburn. Another plausible explanation for heartburn in achalasia is that stasis and fermentation of food in the esophagus produces lactic acid. ${ }^{42}$ Mechanism of chest pain in achalasia is largely unknown. Interestingly, chest pain does not respond as well to medical/surgical treatment of achalasia as dysphagia. Studies also show that chest pain is more common in patients with types II and III than type I achalasia. ${ }^{39}$ Etiology of esophageal "angina like" pain (pressure, squeeze and tightness) and heartburn (burning sensation in the retrosternal region) resistant to adequate acid suppression therapy remains elusive. In the era of over the counter proton pump inhibitor (PPI) drugs, in majority of patients seen by physicians, heartburn and esophageal pain are not related to acid reflux. Prolonged manometry studies (that measures only circular muscle contraction) in late 1980's and early 1990's excluded esophageal spasm as the cause of "angina like" pain. Current thinking is that esophageal pain and PPI resistant heartburn are related to hypersensitivity of the esophagus (hypersensitivity to acid, esophageal distension and other physiological stimuli). ${ }^{43}$ However, prolonged manometry and US imaging studies found a close temporal correlation between sustained (prolonged) contraction of the longitudinal muscle and esophageal pain $^{44}$ (mean duration of contraction 70 seconds) as well as heartburn $^{45}$ (mean contraction duration 30-50 seconds) in approximately two-thirds of the symptom episodes (Fig. 7). Latter raises the possibility that longitudinal muscle spasm of the esophagus may actually be the cause of esophageal pain and PPI resistant heartburn. It may be that the longitudinal muscle spasm is the cause of heartburn and chest pain in achalasia esophagus. Since neither pneumatic dilation nor Heller myotomy impact longitudinal muscle of the esophagus, it is not surprising that chest pain does not respond to treatments.

Prolonged US imaging studies to record longitudinal muscle contraction are not practical because equipment is expensive and data analysis are cumbersome. Alternative strategies are needed to demonstrate the cause and effect relationship between longitudinal muscle spasm and esophageal pain. One such possibility is prolonged ambulatory HRM that can detect cranial lift of the LES as a marker of longitudinal muscle contraction. Feasibility of such methodology has recently been demonstrated ${ }^{46}$ and future studies need to confirm the cause and effect relationship between prolonged longitudinal muscle contraction and esophageal pain.

How does prolonged contraction of the longitudinal muscle

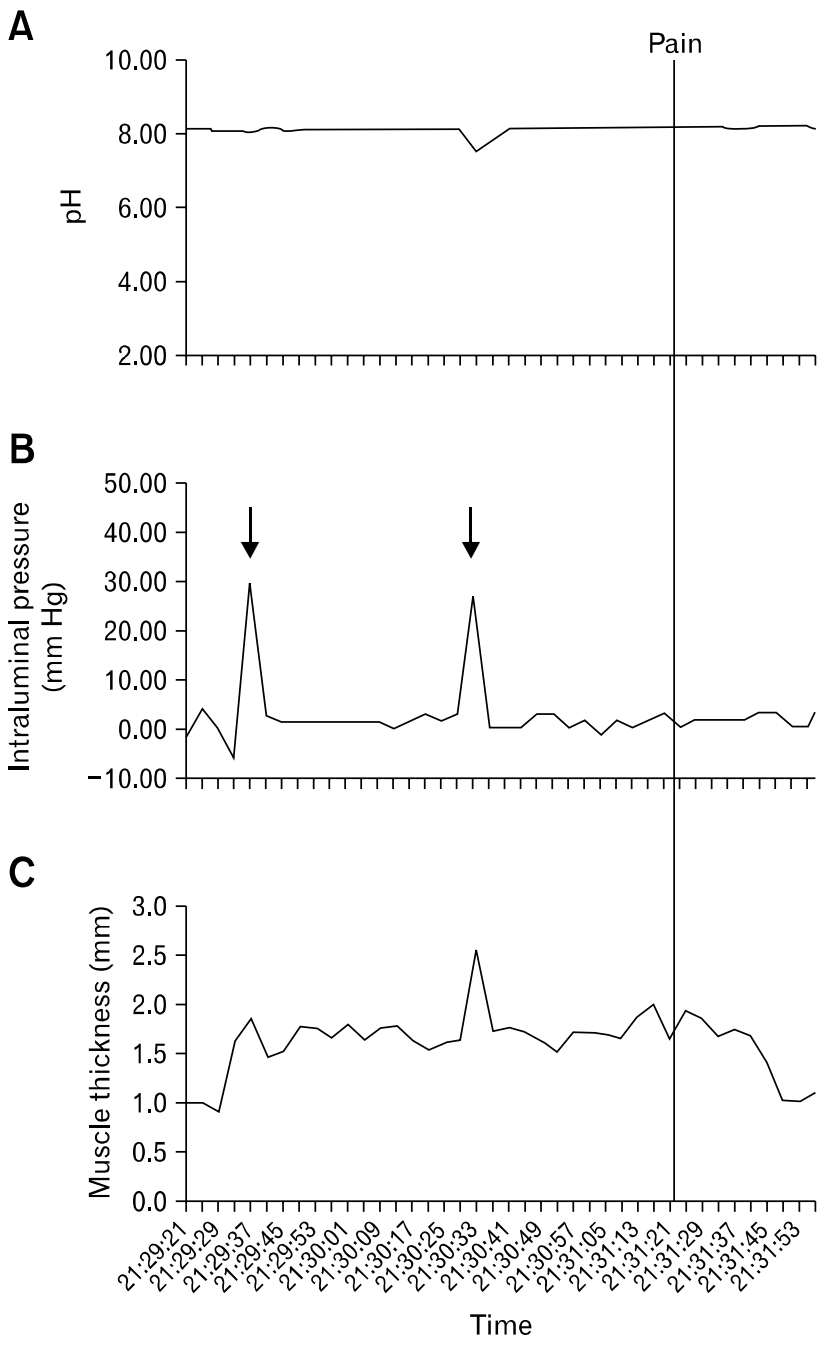

Figure 7. Sustained esophageal contraction associated with chest pain. Esophageal pH (A), distal esophageal pressure (B) and esophageal smooth muscle thickness $(\mathrm{C})$ are shown during a 2.5 -minute recorded interval. The onset of chest pain is depicted by the vertical line (time $=0$ ). The onset of sustained esophageal contraction (SEC) occurs approximately 120 seconds before the onset of pain. The pressure record shows 2 small contractions (arrows) that are accompanied by brief increases in esophageal muscle thickness during the SEC. Adapted from Balaban et al. $^{44}$

cause esophageal pain/heartburn? It is well known that blood flows into the myocardium during diastole because muscle contraction during systole constricts blood vessels and restricts entry of blood into the myocardium. A similar phenomenon has been found in the stomach. ${ }^{47}$ Recent studies show that during swallow-induced esophageal contractions (primary peristalsis) there is almost complete cessation of esophageal wall perfusion (Fig. 8). ${ }^{48,49}$ Remember, during peristalsis circular and longitudinal muscle contract 
A

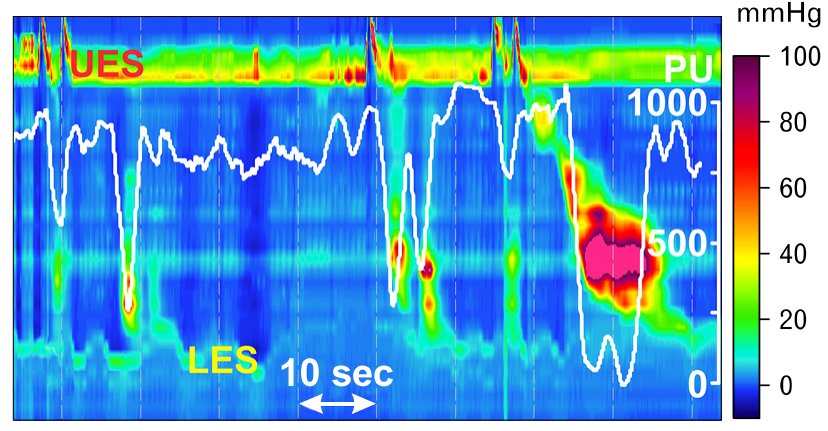

C

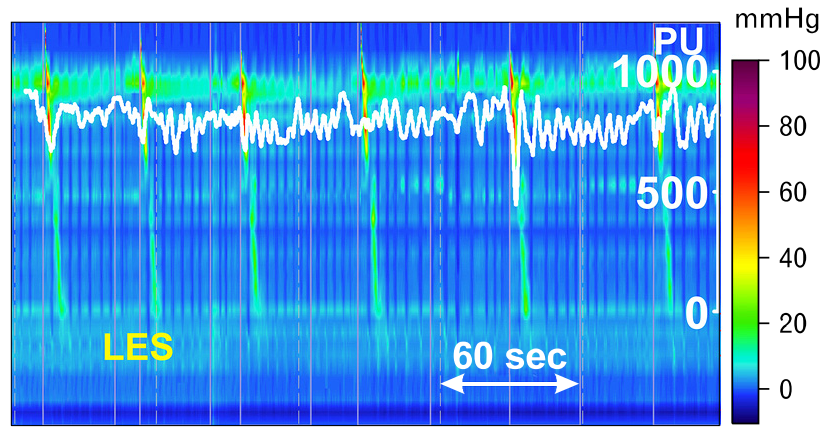

B

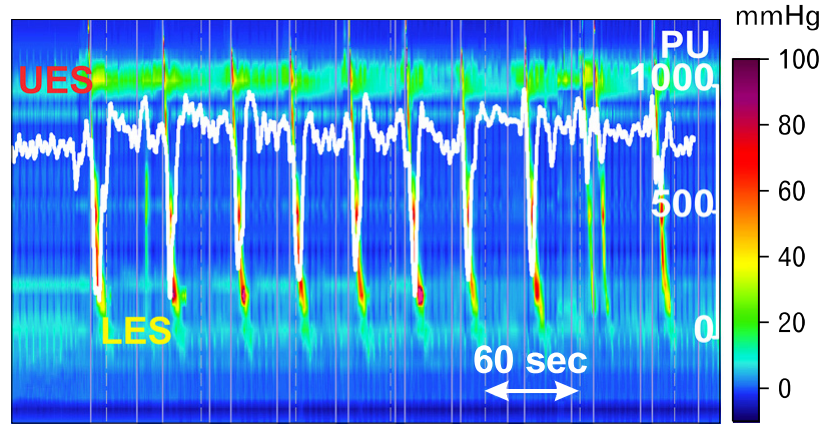

Figure 8. Esophageal wall perfusion during swallow induced esophageal contractions. Doppler perfusion tracings are superimposed white line on the high-resolution manometry (HRM). Doppler transducer was taped to the Bravo $\mathrm{pH}$ capsule which was anchored 4 to $6 \mathrm{~cm}$ above the lower esophageal sphincter. (A) It showed the fall in laser Doppler perfusion with each swallow induced esophageal contraction. Amplitude and duration of perfusion record is related to the amplitude and duration of esophageal contraction. (B) It shows the effect of wet swallows induced esophageal wall contraction on the reduction in esophageal wall perfusion. (C) It shows the effect of atropine on the laser Doppler perfusion during wet swallow. Note, atropine reduced the esophageal contraction amplitude and reduction in blood perfusion with esophageal contractions. UES, upper esophageal sphincter; LES, lower esophageal sphincter. Adapted from Jiang et al. ${ }^{48}$

simultaneously. Transient LES relaxation that is associated with selective longitudinal muscle contraction of the esophagus is also associated with significant reduction in esophageal wall perfusion. ${ }^{48}$ These observations raise the possibility that prolonged contractions of longitudinal muscles of the esophagus could lead to ischemia of the esophageal wall and similar to myocardial pain, "angina like" pain of esophageal origin may be the result of esophageal wall ischemia. It is feasible to measure esophageal wall perfusion for extended periods using laser Doppler flowmetry and future studies need to explore the temporal correlation between esophageal wall ischemia and spontaneous esophageal pain and heartburn.

\section{Summary and Future Directions}

Longitudinal muscles of the muscularis propria comprise 50 percent of the muscle mass of the esophagus and have not been as well studied as the circular muscles. Advances in esophageal manometry techniques, i.e., HRM and topographical color plots to visualize pressures have made it easier to study circular muscle function of the esophagus. In the currently used format, HRM and Chicago classification does not reveal any information about the longitudinal muscle function. Based on the studies conducted during last 2 decades, it is clear that longitudinal muscles of the esophagus play an important role in the physiology as well as pathophysiology of the esophageal sensory and motility disorders. It is highly likely that the etiology of dysphagia and esophageal pain ( 2 cardinal symptoms of esophagus) are linked to the longitudinal muscle dysfunction. Therefore it is imperative to improve diagnostic abilities to study longitudinal muscle function in health and esophageal motor disorders. Ambulatory HRM is one such important modality, the feasibility of which has been demonstrated recently. ${ }^{46}$ Relationship between sustained/prolonged longitudinal muscle contraction and ischemia of the esophageal 
wall in the genesis of esophageal pain requires further exploration. As true for all major advances, development of novel and user friendly techniques are required to improve understanding of the role of longitudinal muscles in the pathophysiology and treatment of esophageal sensory and motor disorders. Longitudinal muscle could be an important target for novel pharmacologic therapeutics to treat sensory and motor dysfunction of the esophagus.

\section{References}

1. Bredenoord AJ, Fox M, Kahrilas PJ, et al. Chicago classification criteria of esophageal motility disorders defined in high resolution esophageal pressure topography. Neurogastroenterol Motil 2012; 24(suppl 1):57-65.

2. Sugarbaker DJ, Rattan S, Goyal RK. Swallowing induces sequential activation of esophageal longitudinal smooth muscle. Am J Physiol 1984;247(5 Pt 1):G515-G519.

3. Pouderoux P, Lin S, Kahrilas PJ. Timing, propagation, coordination, and effect of esophageal shortening during peristalsis. Gastroenterology 1997;112:1147-1154.

4. Edmundowicz SA, Clouse RE. Shortening of the esophagus in response to swallowing. Am J Physiol 1991;260(3 Pt 1):G512-G516.

5. Dodds WJ, Stewart ET, Hodges D, Zboralske FF. Movement of the feline esophagus associated with respiration and peristalsis. An evaluation using tantalum markers. J Clin Invest 1973;52:1-13.

6. Miller LS, Liu JB, Colizzo FP, et al. Correlation of high-frequency esophageal ultrasonography and manometry in the study of esophageal motility. Gastroenterology 1995;109:832-837.

7. Mittal RK, Liu J, Puckett JL, et al. Sensory and motor function of the esophagus: lessons from ultrasound imaging. Gastroenterology 2005; 128:487-497.

8. Lee Y, Whiting J, Roberston E, Derakhshan M, Smith D, McColl $\mathrm{K}$. Measuring movement and location of the gastroesophageal junction: research and clinical implications. Scand J Gastroenterol 2013; 48:401-411.

9. Clouse RE, Staiano A. Topography of the esophageal peristaltic pressure wave. Am J Physiol 1991;261(4 Pt 1):G677-G684.

10. Mittal RK, Padda B, Bhalla V, Bhargava V, Liu J. Synchrony between circular and longitudinal muscle contractions during peristalsis in normal subjects. Am J Physiol Gastrointest Liver Physiol 2006; 290:G431-G438.

11. Yamamoto Y, Liu J, Smith TK, Mittal RK. Distension-related responses in circular and longitudinal muscle of the human esophagus: an ultrasonographic study. Am J Physiol 1998;275(4 Pt 1):G805G811.

12. Babaei A, Bhargava V, Korsapati H, Zheng WH, Mittal RK. A unique longitudinal muscle contraction pattern associated with transient lower esophageal sphincter relaxation. Gastroenterology 2008; 134:1322-1331.

13. Pandolfino JE, Zhang QG, Ghosh SK, Han A, Boniquit C, Kahrilas PJ. Transient lower esophageal sphincter relaxations and reflux: mechanistic analysis using concurrent fluoroscopy and high-reso- lution manometry. Gastroenterology 2006;131:1725-1733.

14. Leslie E, Bhargava V, Mittal RK. A novel pattern of longitudinal muscle contraction with subthreshold pharyngeal stimulus: a possible mechanism of lower esophageal sphincter relaxation. Am J Physiol Gastrointest Liver Physiol 2012;302:G542-G547.

15. Vegesna AK, Chuang KY, Besetty R, et al. Circular smooth muscle contributes to esophageal shortening during peristalsis. World J Gastroenterol 2012;18:4317-4322.

16. Pal A, Brasseur JG. The mechanical advantage of local longitudinal shortening on peristaltic transport. J Biomech Eng 2002;124:94-100.

17. Jiang $Y$, Bhargava V, Mittal RK. Mechanism of stretch-activated excitatory and inhibitory responses in the lower esophageal sphincter. Am J Physiol Gastrointest Liver Physiol 2009;297:G397-G405.

18. Abrahao L Jr, Bhargava V, Babaei A, Ho A, Mittal RK. Swallow induces a peristaltic wave of distension that marches in front of the peristaltic wave of contraction. Neurogastroenterol Motil 2011;23:201207.e110.

19. Gonsalves N, Yang GY, Doerfler B, Ritz S, Ditto AM, Hirano I. Elimination diet effectively treats eosinophilic esophagitis in adults; food reintroduction identifies causative factors. Gastroenterology 2012;142:1451-1459.e1.

20. Furuta GT, Liacouras CA, Collins MH, et al. Eosinophilic esophagitis in children and adults: a systematic review and consensus recommendations for diagnosis and treatment. Gastroenterology 2007; 133:1342-1363.

21. Korsapati H, Babaei A, Bhargava V, Dohil R, Quin A, Mittal RK. Dysfunction of the longitudinal muscles of the oesophagus in eosinophilic oesophagitis. Gut 2009;58:1056-1062.

22. Sugarbaker DJ, Rattan S, Goyal RK. Mechanical and electrical activity of esophageal smooth muscle during peristalsis. Am J Physiol 1984;246(2 Pt 1):G145-G150.

23. Saha JK, Hirano I, Goyal RK. Biphasic effect of SNP on opossum esophageal longitudinal muscle: involvement of cGMP and eicosanoids. Am J Physiol 1993;265(2 Pt 1):G403-G407.

24. Zhang Y, Paterson WG. Nitric oxide contracts longitudinal smooth muscle of opossum oesophagus via excitation-contraction coupling. J Physiol 2001;536(Pt 1):133-140.

25. Crist J, Gidda J, Goyal RK. Role of substance P nerves in longitudinal smooth muscle contractions of the esophagus. Am J Physiol 1986;250(3 Pt 1):G336-G343.

26. Sifrim D, Lefebvre R. Role of nitric oxide during swallow-induced esophageal shortening in cats. Dig Dis Sci 2001;46:822-830.

27. Dodds WJ, Stef JJ, Stewart ET, Hogan WJ, Arndorfer RC, Cohen EB. Responses of feline esophagus to cervical vagal stimulation. Am J Physiol 1978;235:E63-E73.

28. White RJ, Zhang Y, Morris GP, Paterson WG. Esophagitis-related esophageal shortening in opossum is associated with longitudinal muscle hyperresponsiveness. Am J Physiol Gastrointest Liver Physiol 2001;280:G463-G469.

29. Bhalla V, Liu J, Puckett JL, Mittal RK. Symptom hypersensitivity to acid infusion is associated with hypersensitivity of esophageal contractility. Am J Physiol Gastrointest Liver Physiol 2004;287:G65G71.

30. Paterson WG, Miller DV, Dilworth N, Assini JB, Lourenssen S, Blennerhassett MG. Intraluminal acid induces oesophageal shortening via capsaicin-sensitive neurokinin neurons. Gut 2007;56: 
1347-1352.

31. Liu H, Miller DV, Lourenssen S, Wells RW, Blennerhassett MG, Paterson WG. Proteinase-activated receptor-2 activation evokes oesophageal longitudinal smooth muscle contraction via a capsaicin-sensitive and neurokinin-2 receptor-dependent pathway. Neurogastroenterol Motil 2010;22:210-216.e67.

32. Korsapati H, Babaei A, Bhargava V, Mittal RK. Cholinergic stimulation induces asynchrony between the circular and longitudinal muscle contraction during esophageal peristalsis. Am J Physiol Gastrointest Liver Physiol 2008;294:G694-G698.

33. Jung HY, Puckett JL, Bhalla V, et al. Asynchrony between the circular and the longitudinal muscle contraction in patients with nutcracker esophagus. Gastroenterology 2005;128:1179-1186.

34. Korsapati H, Bhargava V, Mittal RK. Reversal of asynchrony between circular and longitudinal muscle contraction in nutcracker esophagus by atropine. Gastroenterology 2008;135:796-802.

35. Kim HS, Park H, Lim JH, et al. Morphometric evaluation of oesophageal wall in patients with nutcracker oesophagus and ineffective oesophageal motility. Neurogastroenterol Motil 2008;20:869-876.

36. Hong SJ, Bhargava V, Jiang Y, Denboer D, Mittal RK. A unique esophageal motor pattern that involves longitudinal muscles is responsible for emptying in achalasia esophagus. Gastroenterology 2010;139:102-111.

37. Pandolfino JE, Kwiatek MA, Nealis T, Bulsiewicz W, Post J, Kahrilas PJ. Achalasia: a new clinically relevant classification by high-resolution manometry. Gastroenterology 2008;135:1526-1533.

38. Rohof WO, Hirsch DP, Kessing BF, Boeckxstaens GE. Efficacy of treatment for patients with achalasia depends on the distensibility of the esophagogastric junction. Gastroenterology 2012;143:328-335.

39. Rohof WO, Salvador R, Annese V, et al. Outcomes of treatment for achalasia depend on manometric subtype. Gastroenterology 2013;
144:718-725.

40. Eckardt VF, Stauf B, Bernhard G. Chest pain in achalasia: patient characteristics and clinical course. Gastroenterology 1999;116:13001304.

41. Spechler SJ, Souza RF, Rosenberg SJ, Ruben RA, Goyal RK. Heartburn in patients with achalasia. Gut 1995;37:305-308.

42. Richter JE. Achalasia - an update. J Neurogastroenterol Motil 2010; 16:232-242.

43. Miwa H, Kondo T, Oshima T, Fukui H, Tomita T, Watari J. Esophageal sensation and esophageal hypersensitivity - overview from bench to bedside. J Neurogastroenterol Motil 2010;16:353-362.

44. Balaban DH, Yamamoto Y, Liu J, et al. Sustained esophageal contraction: a marker of esophageal chest pain identified by intraluminal ultrasonography. Gastroenterology 1999;116:29-37.

45. Pehlivanov N, Liu J, Mittal RK. Sustained esophageal contraction: a motor correlate of heartburn symptom. Am J Physiol Gastrointest Liver Physiol 2001;281:G743-G751.

46. Mittal RK, Karstens A, Leslie E, Babaei A, Bhargava V. Ambulatory high-resolution manometry, lower esophageal sphincter lift and transient lower esophageal sphincter relaxation. Neurogastroenterol Motil 2012;24:40-46.e42.

47. Livingston EH, Howard TJ, Garrick TR, Passaro EP Jr, Guth PH. Strong gastric contractions cause mucosal ischemia. Am J Physiol 1991;260(3 Pt 1):G524-G530.

48. Jiang Y, Bhargava V, Kim YS, Mittal RK. Esophageal wall blood perfusion during contraction and transient lower esophageal sphincter relaxation in humans. Am J Physiol Gastrointest Liver Physiol 2012;303:G529-G535.

49. Mittal RK, Bhargava V, Lal H, Jiang Y. Effect of esophageal contraction on esophageal wall blood perfusion. Am J Physiol Gastrointest Liver Physiol 2011;301:G1093-G1098. 